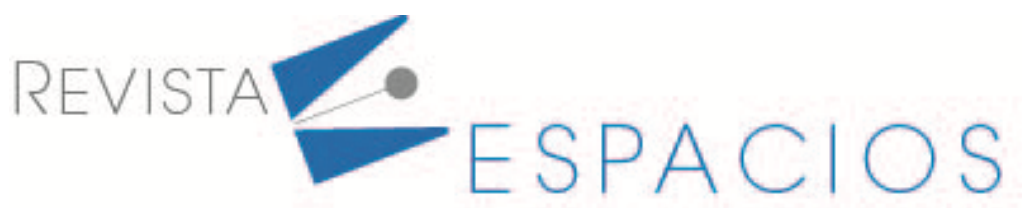

\title{
Diseño de un sistema de información gerencial para apoyar la prevención del virus papiloma humano
}

\section{Design of a management information system to support the prevention of the human papillomavirus}

\author{
PUELLO, Plinio ${ }^{1}$ \\ SALGADO, Orley ${ }^{2}$ \\ MONROY, Martín E. ${ }^{3}$
}

\section{Resumen}

El Centro de Atención Primaria (CAP) ofrece servicios médicos y realiza campañas de detección y prevención del virus del papiloma humano (VPH). Se diseñó un sistema de información gerencial para apoyar la prevención del VPH, aplicando la metodología de componentes estructurada en tres fases. Como resultado se obtuvo el modelo de negocio del CAP, se identificaron los desafíos que debe afrontar, se describieron los componentes organizacional, administrativo y tecnológico; y se diseñó la solución tecnológica.

Palabras clave: sistemas de información gerencial, virus del papiloma humano, modelo de negocio, prevención.

\begin{abstract}
The Primary Care Center (PCC) offers medical services and conducts human papillomavirus (HPV) detection and prevention campaigns. A management information system was designed to support the prevention of HPV, applying the methodology of components structured in three phases. As a result, the PCC business model was obtained, the challenges it must face were identified, the organizational, administrative and technological components were described; and the technological solution was designed.
\end{abstract}

key words: management information systems, human papillomavirus, business model, prevention

\section{Introducción}

Colombia es una nación con más de 47 millones de habitantes, cuya población está creciendo y envejeciendo. Su economía está en desarrollo y el sistema de salud ha venido en un constante progreso bajo el enfoque de cobertura universal. Los hábitos de estilo de vida están cambiando con la disminución de la actividad física, las tasas de fertilidad y el aumento de la obesidad. Esto ha influido en el aumento del cáncer en Colombia (de Vries, et al., 2018). Sólo para el caso del cáncer cervical las estimaciones netas de supervivencia son similares a las de

\footnotetext{
${ }^{1}$ Profesor de la Universidad de Cartagena. Programa de Ingeniería de Sistemas. Facultad de Ingeniería. Ingeniero de Sistemas, PhD.(c) en Ingeniería. ppuellom@unicartagena.edu.co

2 Estudiante del programa de ingeniería de sistemas. Universidad de Cartagena. osalgadoo@unicartagena.edu.co

${ }^{3}$ Profesor de la Universidad de Cartagena. Programa de Ingeniería de Sistemas. Facultad de Ingeniería. Ingeniero de Sistemas, MSc. en Ciencias Computacionales, PhD. en Ingeniería Telemática.mmonroyr@unicartagena.edu.co
} 
los EE. UU., 59.3\% en Colombia versus 62.3\% en los EE. UU. (de Vries, - et al., 2018). En el país el cáncer es una enfermedad de creciente relevancia, los patrones de incidencia están cambiando, con disminuciones en cánceres como la neoplasias de estómago y cervicales, y aumentos en el cáncer de mama, colorrectal y de próstata (Piñeros et al., 2013). En 2012 se estimaron casi 1.1 millones de nuevos casos de cáncer al año, con variaciones importantes en las tasas entre los distintos países. Los cánceres de estómago y cuello uterino ocuparon puestos altos en los países menos desarrollados. Para el año 2030 se espera un incremento del $66 \%$ en los casos de cáncer(Bray \& Piñeros, 2016).

El cáncer cervicouterino o cáncer de cuello uterino es causado por el virus del papiloma humano (VPH). Los factores más comunes de contagio son: las relaciones sexuales tempranas, la promiscuidad tanto de la mujer como del hombre, mujeres con alto número de embarazos, el parto en edades tempranas, la depresión del sistema inmunológico, el uso prolongado de anticonceptivos y la mala nutrición, aumentan la probabilidad de desarrollar una infección de VPH (Contreras-González et al., 2017). Hay 200 tipos de VPH y al menos 40 pueden infectar el área anogenital. Los tipos de VPH se clasifican en subtipos cutáneos y mucosos. Sin embargo, aún no se recomienda la evaluación en hombres para el VPH, porque son menos propensos al contraer el virus. También las relaciones de hombres de alto riesgo que tienen sexo con hombres (HSH) y hombres VIH positivos, lo que justifica la adopción de detección adicional (Vives et al., 2020).

El cáncer de cuello uterino es el cuarto cáncer más común en mujeres en el mundo, alrededor del $85 \%$ del mundo ocurre en países de bajos y medianos ingresos. Representa el $12 \%$ de todos los cánceres ocurridos en mujeres debido al VPH (Ferlay et al., 2013). Las personas se infectan con el VPH al menos una vez en la vida, y hay una alta tasa de detección en jóvenes sexualmente activos, cuyos factores de riesgo incluyen: una edad temprana en la primera relación sexual, el número de parejas sexuales y el sexo oral (Vives et al., 2020). El cáncer de cuello uterino es un problema de salud importante a pesar de la introducción de las vacunas contra el VPH. Dentro de 20 a 30 años es probable que haya una reducción de $70 \%$ a $80 \%$ en la incidencia de cáncer cervical en mujeres vacunadas, que disminuiría el porcentaje de falsos negativos y reduciría la incidencia de cáncer cervical, salvando más vidas de mujeres (Tjalma et al., 2017).

El cáncer cervical o de cuello uterino es una enfermedad de los grupos socioeconómicos más bajos. Colombia ha implementado una gran reforma en su sistema de salud, con el objetivo de proporcionar un acceso igualitario a todos los grupos socioeconómicos (Guerrero et al., 2011), logrando un aumento importante en la cobertura y disponibilidad de servicios de salud para la población y reflejando una tendencia decreciente y prometedora con respecto a las desigualdades (Piñeros et al., 2013). Sin embargo, investigaciones más recientes mostraron una tendencia creciente en las desigualdades educativas y en la mortalidad general por cáncer femenino en Colombia (de Vries, et al., 2018).

Por su parte, la Universidad de Cartagena como entidad pública, para el cumplimiento de sus objetivos misionales relacionados con la proyección social, tiene una división administrativa denominada Sección de Salud y Seguridad Laboral, que cuenta con el programa de Enfermería, cuya misión consiste en ofrecer servicios de salud al individuo, la familia y en entorno, mediante la promoción de la salud, el auto cuidado y la práctica de estilos de vida saludables. Además, ofrece servicios médicos, de enfermería y educación de salud, apoyando el proceso de formación de profesionales de Enfermería con sentido crítico y ético, para el desempeño en los procesos de intervención a la persona, la familia y la comunidad. Estos servicios se encuentran disponibles para toda la comunidad en el Centro de Atención Primaria (CAP de San Pablo), e incluyen: el programa ampliado de inmunizaciones PAl, el programa para la atención en planificación familiar a hombres y mujeres, consulta de enfermería, consulta de medicina, consulta de psicología y citología entre otros.

El servicio de citología consiste en la toma de una muestra de las células de la vagina y del cuello uterino. Es una prueba que sirve para detectar el VPH y se debe practicar cada año en la revisión ginecológica, a mujeres mayores 
de 16 años que hayan tenido relaciones sexuales. Actualmente el CAP lleva un registro manual de la información clínica de cada uno de sus pacientes, y su sistema de información no incluye el apoyo de tecnologías de la información y las comunicaciones. Esto dificulta en forma representativa los procesos de atención y la toma de decisiones tendientes a mejorar los servicios que presta, sobre todo para los programas de detección basados en citología, porque exigen múltiples visitas para muestra de citología vaginal, colposcopia, biopsia y tratamientos.

Aunque vacunarse contra el VPH puede ayudar a prevenir estos problemas de salud, si se tiene en cuenta que las mujeres cribadas positivamente a menudo deben visitar centros de salud distantes; $y$ si se diagnostica cáncer invasivo, los pacientes con frecuencia tienen que buscar tratamiento en centros de salud aún más distantes (Murillo et al., 2010). Por lo cual, si se crea un programa de detección de cáncer de cuello uterino sería una gran empresa para el sistema de salud pública, salvando así a muchas personas si se detecta antes, dándoles tranquilidad al saber que no tienen enfermedades, (Tjalma et al., 2017). En este orden de ideas, se propone el diseño de un sistema de información gerencial para apoyar la prevención del virus papiloma humano, como parte del sistema de salud pública en el CAP de la Universidad de Cartagena.

El artículo se estructura de la siguiente manera: a continuación, se describe la metodología usada para diseñar el sistema de información gerencial. Luego se explican los resultados obtenidos en cada una de las fases de la metodología aplicada, posteriormente se plantea la discusión sobre los resultados y finalmente se presentan las conclusiones del trabajo.

\section{Metodología}

Para el diseño del sistema de información gerencial propuesto, se realizaron las actividades que comprende la metodología de componentes (Laudon \& Laudon, 2014), que incluye las siguientes fases:

\subsection{Fase de análisis y desafíos del negocio}

Esta fase tiene como fin recopilar información sobre el contexto del problema para identificar las necesidades del entorno, haciendo uso de técnicas de recolección de información, como entrevistas aplicadas a fuentes primarias, representadas por los funcionarios del CAP, quienes poseen el conocimiento y la experiencia sobre lo que sucede al interior de la organización. De igual forma se utilizan fuentes secundarias aplicando técnicas de recolección de información como la revisión bibliográfica, haciendo uso de diferentes bases de datos bibliográficas. Como técnica de análisis de la información se utiliza la elaboración del modelo de negocio del contexto de problema (Amit et al., 2011), identificando los procesos, sus actores y responsabilidades dentro de la organización.

\subsection{Fase de descripción de componentes}

El propósito de esta fase, como su nombre lo indica, consiste en describir los componentes administrativo, organizativo y tecnológico, que hacen parte de todo sistema de información gerencial, en función de lo que se tiene y lo que se requiere para brindar una solución al problema objeto de análisis. Esto se logra como resultado del análisis detallado de la información recolectada en la fase inmediatamente anterior, y sirve de insumo para la definición del diseño del sistema de información gerencial para apoyar la prevención del VPH.

\subsection{Fase de planteamiento de una solución tecnológica}

En esta fase se diseña la solución tecnológica, representada por el sistema de información gerencial, que debe satisfacer los desafíos y cumplir con los requisitos establecidos. El cumplimiento de esta fase implica la realización de un proceso de ingeniería de software. Teniendo en cuenta el alcance de la investigación sólo se presenta el modelo de diseño del sistema de información gerencial propuesto. Para la representación de los modelos se 
utiliza el Lenguaje Unificado de Modelado UML (Object Management Group, 2015) y el enfoque de descripción de arquitectura denominado 4+1 (Kruchten, 1995).

\section{Resultados}

Los resultados se presentan en función de cada una de las fases de la metodología. Inicialmente se describe el modelo de negocio, construido a partir del análisis realizado sobre la documentación recolectada. Además, se hace un análisis sobre los trabajos relacionados identificados en la revisión bibliográfica. Esta información es usada como insumo para identificar los desafíos del negocio y los requisitos que debe cumplir el sistema de información gerencial. Posteriormente se describe cada componente del sistema de información gerencial, comenzando por el componente administrativo, luego el componente organizativo y a continuación el componente tecnológico. Finalmente se explica el diseño del sistema de información propuesto, resaltando la definición del modelo de diseño y las vistas arquitectónicas del sistema.

\subsection{Análisis y desafíos del negocio}

La aplicación de la técnica análisis de modelo de negocio condujo a determinar que el CAP ofrece diferentes servicios médicos, entre los cuales se encuentra el servicio de citología, como se observa en el modelo de dominio (ver Figura 1). Este servicio es programado para cada paciente en una fecha y hora, dentro de los horarios de atención establecidos para este propósito. Requiere de un kit especial que hace parte del inventario de insumos del CAP, el cual es gestionado por la Enfermera. Durante la prestación del servicio de citología la enfermera realiza la prueba, tomando una muestra que es enviada al laboratorio clínico, donde emiten un resultado que puede durar hasta diez días. Cuando se recibe el resultado, el médico realiza el análisis respectivo y emite un diagnóstico que es registrado en la historia clínica del paciente. Luego se cita al paciente para entregarle los resultados. Si no existen novedades la enfermera le explica los resultados y le agenda la cita para la próxima citología. Cuando existen el riesgo de que el paciente sea portador del VPH, se le recomienda hacer una colposcopia directamente en la Entidad Prestadora de Servicio (EPS) en la cual esté afiliado, debido a que el CAP no cuenta con la capacidad necesaria realizarla. Se debe indicar que el CAP como una institución de salud no hace contratos con EPS.

El CAP atiende pacientes que hacen parte de la comunidad universitaria, estudiantes y empleados de la Universidad, y personas del común. Cuenta con un equipo de personal médico conformado por enfermeras y médicos con diferentes especialidades. Además de los servicios médicos, el CAP realiza diferentes tipos de campañas preventivas, tendientes a generar una cultura del autocuidado en las personas. Estas campañas son gestionadas por las enfermeras del CAP. En consecuencia, el análisis realizado condujo a identificar que el sistema cuenta con dos actores: El personal médico y el paciente. Como se observa en el modelo de casos de uso del contexto del problema (ver Figura 2), el personal médico está conformado por las enfermeras y los médicos, mientras que el paciente es un actor pasivo, que puede ser miembro de la comunidad académica, en calidad de estudiante o trabajador de la Universidad, o cualquier persona del común.

También se identificó que en el contexto del problema se realizan los siguientes procesos para que el CAP cumpla con sus propósitos misionales: 1) Atender a los pacientes, 2) gestionar campañas preventivas y 3) Gestionar el inventario de insumos. Cada uno de estos procesos a su vez implica la realización de varias actividades. En el caso de la atención a los pacientes, se requiere gestionar el servicio de citología, gestionar las citas para la prestación de los servicios médicos, realizar la atención al paciente y gestionar su historia clínica. Asimismo, la gestión de las campañas preventivas implica diseñar la campaña, realizar la campaña y analizar los resultados de la ejecución de la campaña. De igual manera, la gestión de los insumos de inventario, consiste en mantener un control sobre los materiales que requiere el CAP, hacer pedidos, registrar las compras y los consumos que he hagan en la ejecución de la prestación de los servicios. 
Figura 1

Modelo de dominio

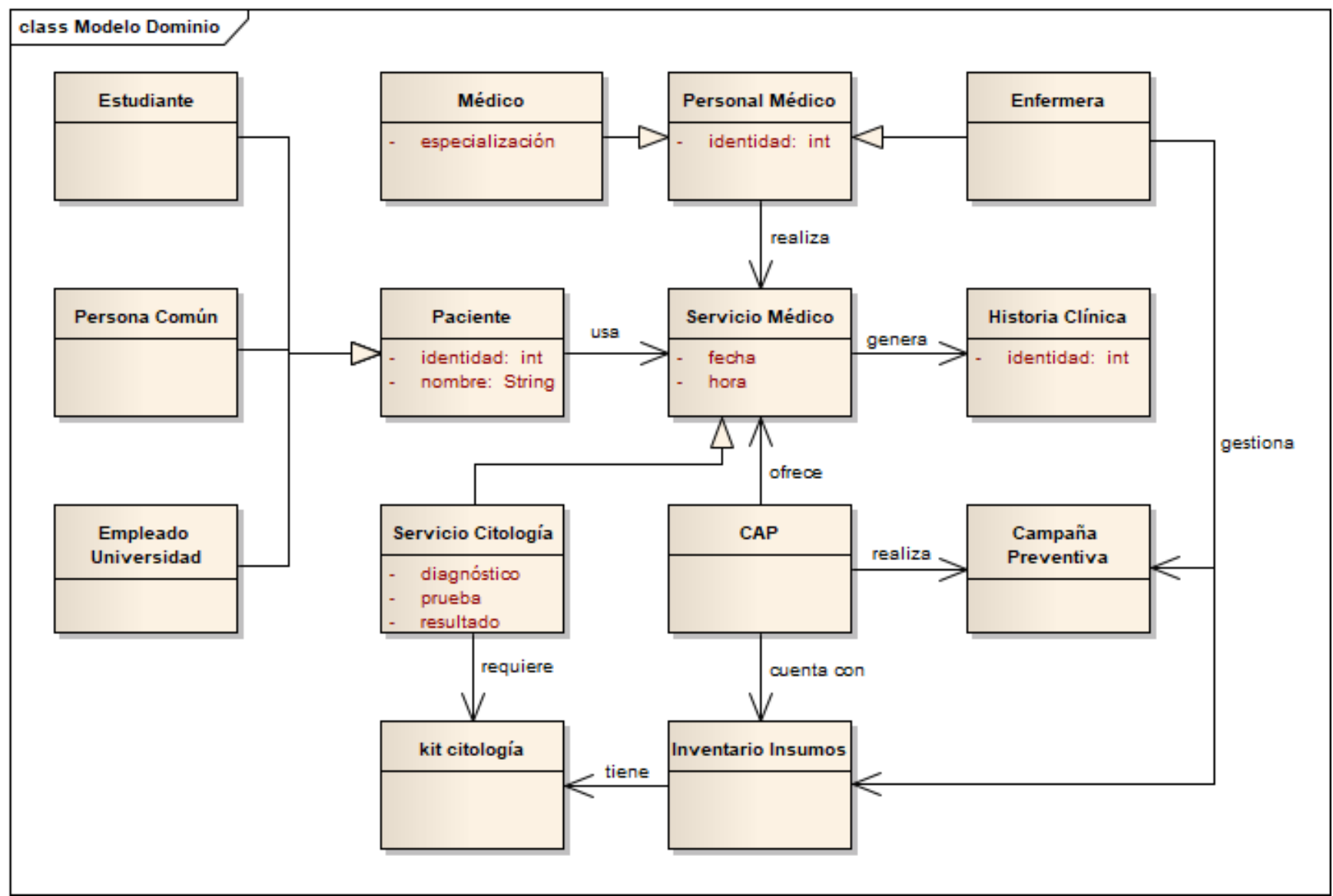

Fuente: De los Autores

Por otra parte, la revisión de la literatura permitió identificar que se ha realizado trabajos de exploración de los requisitos de información del gerente de enfermería, las prácticas de gestión de riesgos y las influencias en la toma de decisiones, al interactuar con un sistema electrónico de gestión de riesgos y notificación de incidentes (Islam et al., 2018). Esto representa una ayuda a los administradores de enfermería y a la atención médica al momento de identificar prácticas ineficaces y cumplir con los requisitos de información del administrador de enfermería. De igual forma, se han descrito los beneficios y posibles consecuencias al integrar las tecnologías de la información y las comunicaciones en los procesos de atención médica (Maass et al., 2008). También se han desarrollado plataformas para apoyar la toma de decisiones en la realización de proyectos de investigación y el desarrollo de tecnologías en el campo de la salud pública, realizando evaluaciones de los proyectos propuestos, con base en modelos de variables (da Rocha et al., 2018). 
Figura 2

Casos de uso contexto del problema

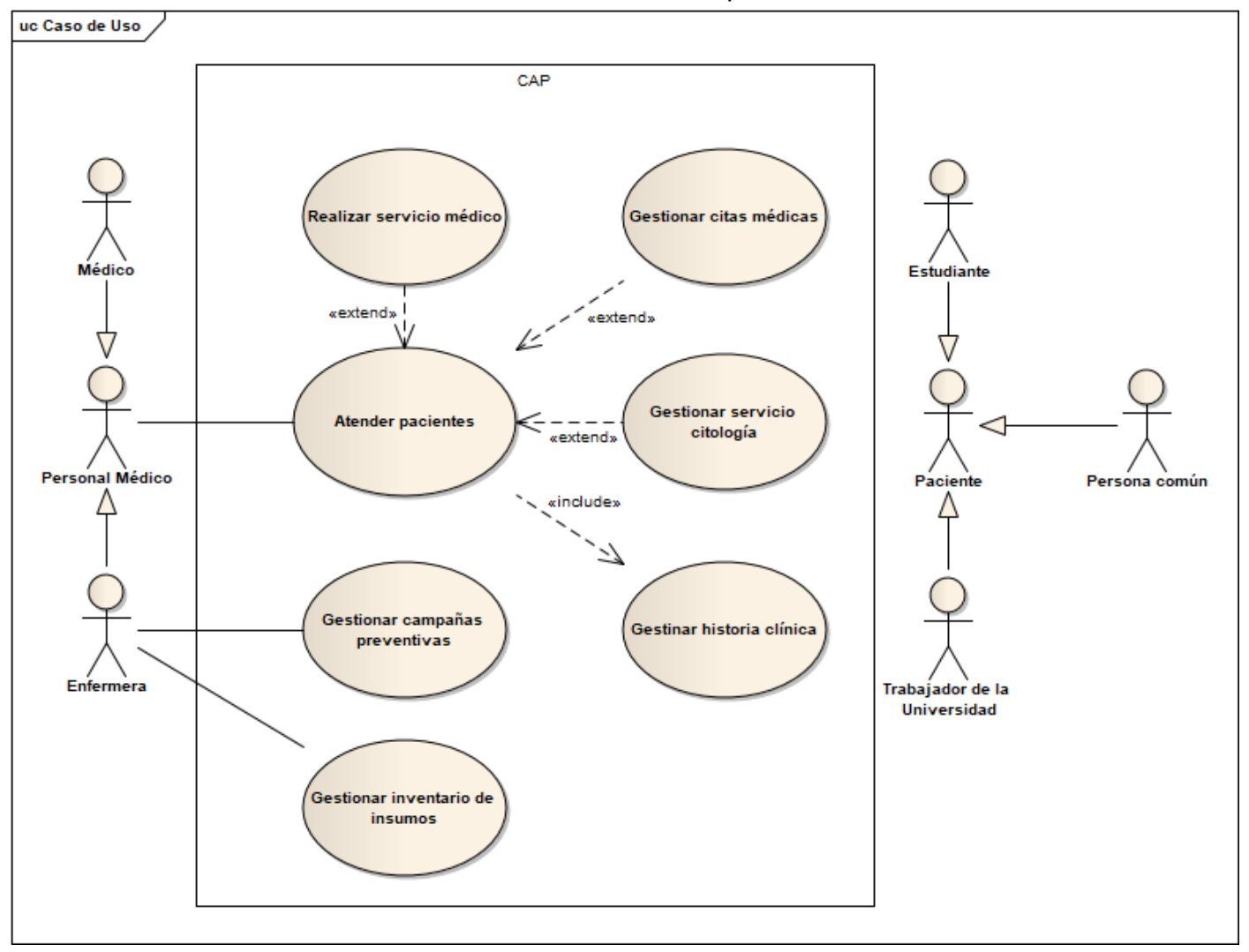

Fuente: De los Autores

Adicionalmente, se identificaron trabajos dirigidos a comprender con mayor precisión el papel de los sistemas de gestión de la información, desde la perspectiva de la salud pública en las unidades de atención primaria (Zhao et al., 2020), confirmando que este tipo de sistemas, no solo cultivan una conciencia de salud y prevención de enfermedades en los actores del contexto de la salud pública, sino que también mejoran la eficiencia en la atención de las instituciones y reducen el número de pacientes que buscan atención médica (Zhao et al., 2020). Si bien todos estos trabajos representan valiosos aportes al estado de la técnica, no se identificó ningún trabajo que proponga concretamente el diseño de un sistema de información gerencial para apoyar la prevención del VPH, lo cual representa el mayor aporte de la presenta investigación.

Para la identificación de los desafíos se analizó la información disponible en el CAP, obteniendo los siguientes resultados. Actualmente el flujo de pacientes usuarios de los servicios médicos ofrecidos por el CAP es muy bajo, incluyendo el servicio de citología. También se encontró que la a historia clínica se registra en archivos físicos, que algunos datos relacionados con la prestación del servicio, como los costos, la información del paciente, entre otros, se registran en una hoja de Excel. Los resultados de las pruebas de laboratorio que llegan en formato digital al correo institucional, se imprimen y se guardan en el archivo físico. De igual forma, la enfermera registra esta información en un archivo de Excel, para tener un seguimiento de las citologías que se han entregado, indicando quien la recibió, cuándo (fecha y hora) y cuál fue el resultado. Con esta información se generan reportes mensuales y anuales, que evidencias los resultados de los servicios ofrecidos por el CAP.

Como se observa en la Figura 3, en el servicio de citología se atendieron 60 pacientes entre abril y diciembre del año 2019, siendo el mes de octubre el de mayor afluencia, con 18 pacientes y noviembre el de menor con un solo paciente. En la mayoría de los meses el flujo no supera las 9 personas. También se identificó que el $72 \%$ de las pacientes que usan el servicio de citología se encuentran en el rango de edad entre los 17 y los 26 años, el 
$17 \%$ están entre los 27 y los 36 años, el $6 \%$ entre los 47 y los 56 , y el $5 \%$ están entre los 47 y los 46 años, como muestra en la - Figura 4 .

Figura 3

Flujo mensual de pacientes

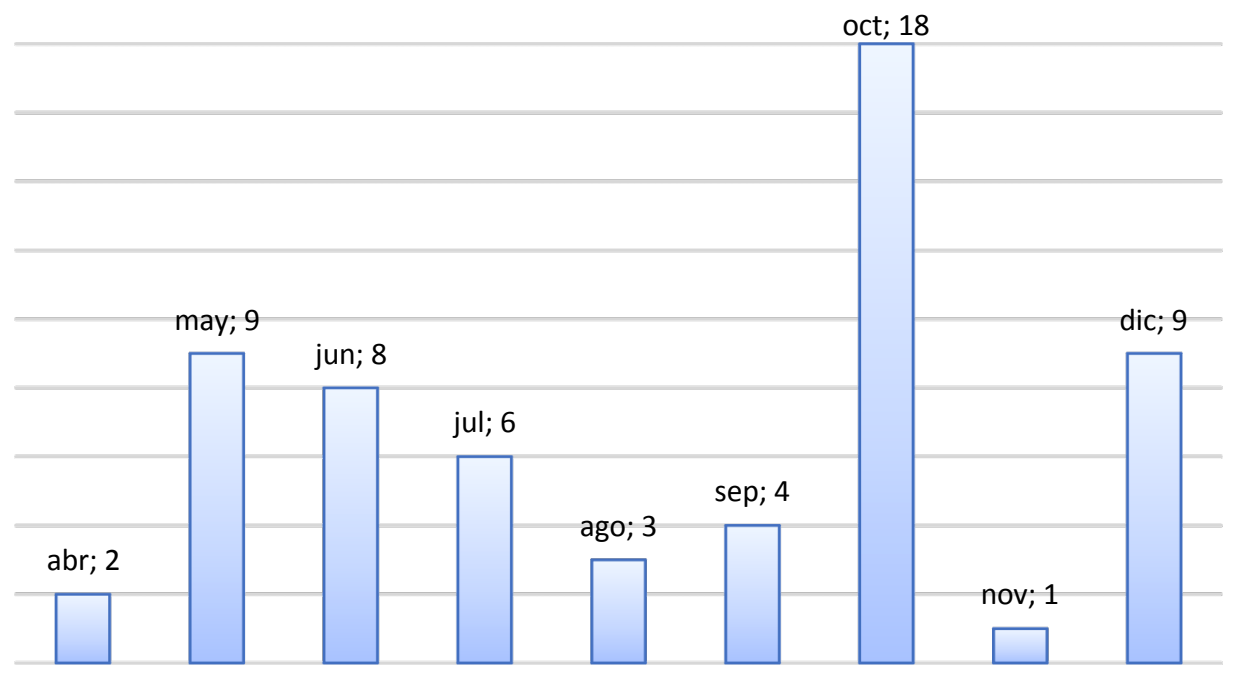

Fuente: De los Autores

Figura 4

Edades de Pacientes Atendidos

47 a 56 años; $6 \%$

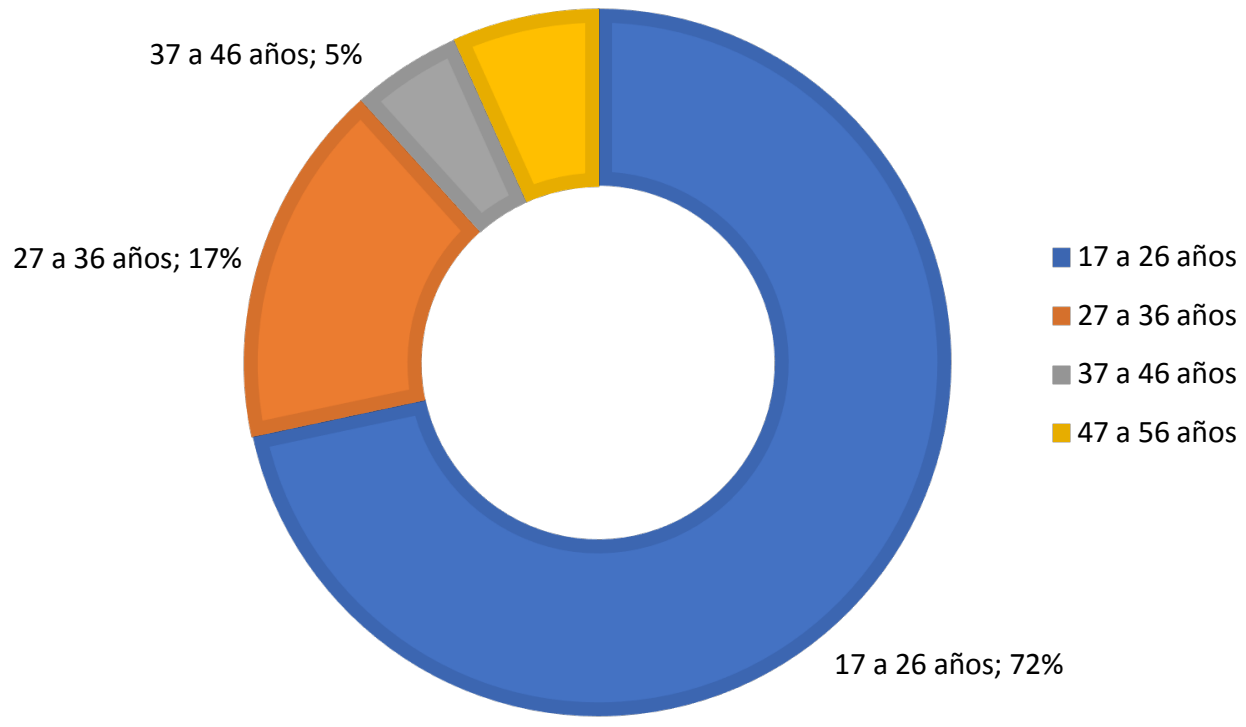

Fuente: De los Autores

En consecuencia, se identificó que los desafíos que debe afronta el CAP son: 1) Generar un mayor impacto en la comunidad ampliando el número de usuarios atendidos y mejorando la calidad de los servicios que ofrece. 2) Sistematizar sus procesos, concretamente los relacionados con la prestación del servicio de citología. Para el 
logro del segundo desafío se establecieron los requisitos funcionales y no funcionales, que se relacionan en la tabla 1 y 2 respectivamente.

Tabla 1

Tabla de Requisitos Funcionales

\begin{tabular}{|c|c|c|c|}
\hline Identificador & Requisito funcional & Descripción & Prioridad \\
\hline RF-01 & $\begin{array}{l}\text { Gestionar historia } \\
\text { clínica }\end{array}$ & $\begin{array}{l}\text { El sistema debe permitir el registro y actualización de la } \\
\text { historia clínica de los pacientes, concretamente los aspectos } \\
\text { relacionados con los servicios de citología. }\end{array}$ & Alta \\
\hline RF-02 & $\begin{array}{l}\text { Gestionar pruebas de } \\
\text { citología }\end{array}$ & $\begin{array}{l}\text { El sistema debe permitir registrar la orden de la prueba de la } \\
\text { historia clínica y sus respectivos resultados, relacionándolos } \\
\text { directamente con la historia clínica de cada paciente. }\end{array}$ & Alta \\
\hline RF-03 & Gestionar citas & $\begin{array}{l}\text { El sistema debe permitir programar y actualizar las citas } \\
\text { médicas para los servicios que presta el CAP, teniendo en } \\
\text { cuenta los horarios de servicio, la disponibilidad de tiempo de } \\
\text { los médicos y enfermeras que prestan los servicios, y } \\
\text { concretamente el servicio de citología. }\end{array}$ & Media \\
\hline RF-04 & $\begin{array}{l}\text { Gestionar Insumos para } \\
\text { pruebas citologías }\end{array}$ & $\begin{array}{l}\text { El sistema debe permitir el registro de ingresos y consumos de } \\
\text { los insumos necesarios para las pruebas de citología. }\end{array}$ & Media \\
\hline RF-05 & $\begin{array}{l}\text { Gestionar campañas } \\
\text { preventivas VPH }\end{array}$ & $\begin{array}{l}\text { El sistema debe permitir la programación y control de } \\
\text { realización de las campañas orientadas a la prevención y } \\
\text { detección del VPH realizadas por el CAP. }\end{array}$ & Media \\
\hline RF-06 & Generar informes & $\begin{array}{l}\text { El sistema debe permitir genera informes que apoyen la toma } \\
\text { de decisiones por parte del personal del CAP. El contenido de } \\
\text { los informes se definirá con base en los datos capturados por } \\
\text { el sistema y su formato será establecido bajo acuerdo con el } \\
\text { personal médico. }\end{array}$ & Baja \\
\hline
\end{tabular}

Fuente: De los autores

Tabla 2

Tabla de Requisitos No Funcionales

\begin{tabular}{|c|c|c|}
\hline Identificador & $\begin{array}{l}\text { Nombre Del Requisito } \\
\text { No Funcional }\end{array}$ & Descripción \\
\hline RNF-01 & Fiabilidad & $\begin{array}{l}\text { El sistema debe evitar la introducción de información errada, mantener un } \\
\text { registro y generación de informes siempre libres de error. }\end{array}$ \\
\hline RNF-02 & Seguridad & $\begin{array}{l}\text { El sistema debe garantizar la integridad y el no repudio de la información, } \\
\text { así como también el acceso a los datos única u exclusivamente por parte } \\
\text { de las personas autorizadas. }\end{array}$ \\
\hline RNF-03 & Disponibilidad & $\begin{array}{l}\text { El sistema debe garantizar un } 97 \% \text { de disponibilidad durante los horarios } \\
\text { de prestación del servicio del CAP. }\end{array}$ \\
\hline RNF-04 & Desempeño & $\begin{array}{l}\text { Los tiempos de respuesta del sistema para las funciones relacionadas con } \\
\text { la captura de datos no será superior a } 3 \text { segundos, y en la generación de } \\
\text { informes no superará los } 30 \text { segundos. }\end{array}$ \\
\hline RNF-05 & Usabilidad & El tiempo de aprendizaje del sistema por usuario debe ser menor a 2 horas. \\
\hline RNF-06 & $\begin{array}{l}\text { Capacidad de } \\
\text { mantenimiento }\end{array}$ & $\begin{array}{l}\text { El sistema debe ser diseñado para crecer en su funcionalidad, de tal } \\
\text { manera que se puedan incorporar componentes que contemplen los } \\
\text { demás servicios prestados por el CAP }\end{array}$ \\
\hline
\end{tabular}

Fuente: De los autores 


\subsection{Descripción de componentes}

Todo sistema de información comprende la organización, la administración y las tecnologías de la información (Laudon \& Laudon, 2014). Un sistema de información debe generar valor para la empresa, representado en una solución organizacional y gerencial, que sirva para afrontar y superar los desafíos impuestos por el entorno. En este orden de ideas, a continuación, se describen cada uno de los componentes organizativo, administrativo y tecnológico del CAP, como resultado de análisis que se hizo de la información recolectada en la fase previa.

\section{Componente Organizativo}

EI CAP está adscrito al programa de Enfermería de la Universidad de Cartagena y ofrece los siguientes servicios: "Programa ampliado de inmunizaciones PAl, programa para la atención en planificación familiar a hombres y mujeres, consulta por enfermería, consulta por medicina, consulta por psicología, detección temprana de alteraciones del crecimiento y desarrollo en el menor de 10 años, detección temprana del cáncer de cuello uterino y el programa de detección temprana de alteraciones del adulto mayor" (Facultad de enfermería, s.f). Para cumplir con estos servicios, el CAP tiene una estructura organizativa representada en tres niveles: La gerencia de nivel superior, que corresponde a la decanatura de la facultad de enfermería, la gerencia de nivel medio bajo la responsabilidad de la enfermera en Jefe, y la parte operativa conformada por el personal médico compuesto por médicos generales, médicos especialistas, médicos practicantes y enfermeras.

La enfermera en jefe es responsable del control sobre el inventario de los insumos, el diseño y programación de campañas de detención y prevención del VPH, la programación de capacitaciones para el personal médico, programación de citas médicas para los pacientes y la gestión de agenda del personal médico. Por su parte, el personal médico es responsable de la prestación de los servicios ofrecidos por el CAP. En consecuencia, se identificaron dos procesos de negocio: El proceso administrativo y el proceso operativo. El primero comprende las siguientes actividades: Gestionar el inventario de insumos, gestionar las campañas de detección y prevención del VPH, gestionar las citas médicas, gestionar la agenda del personal médico, gestionar la agenda de los servicios que se prestan y tomar de decisiones para garantizar la calidad y el mejoramiento de los servicios prestados. El segundo proceso es de carácter operativo y consiste en atender a los pacientes que acuden para recibir los servicios médicos ofrecidos por el CAP. Dentro de las actividades que se desprenden de este proceso están: gestionar la historia clínica de los pacientes, autorizar la realización de pruebas y exámenes de laboratorio.

\section{Componente Administrativo}

La gerencia del CAP, toma decisiones sobre el talento humano, los recursos financieros, los insumos, los recursos tecnológicos y los servicios que ofrece el CAP. Además, formula los planes de acción que proyectan el CAP en el mediano y largo plazo. Con respecto al talento humano se identificó que realiza jornadas de capacitación y define estrategias para optimizar la gestión de la agenda del personal médico. En cuanto a los insumos se estableció que define lineamientos para su adquisición y el control del consumo. En relación con los recursos tecnológicos decide sobre su adquisición y uso dentro del CAP. En lo referente a los servicios, es responsable de diseñar la agenda, controlar las citas de la prestación de los servicios y controlar la calidad de la prestación de los servicios.

Para lograr todas y cada una de estas tareas debe tomar distintas decisiones, relacionadas con cada uno de estos aspectos. Algunas de estas decisiones se relacionan a continuación, para cada una de las dimensiones del sistema de información gerencial: 1) Optimización de la agenda del personal médico, 2) identificar debilidades de formación u oportunidades de prestación de servicio para establecer programas de formación del personal médico, 3) establecer estrategias para mejorar la capacidad de insumos para la realización de las pruebas, 4) modernizar los recursos tecnológicos e involucrarlos en los procesos organizacionales para mejorar la prestación de servicios, 5) Rediseñar las campañas de detección y promoción del VPH para ampliar su cobertura, 6) optimizar el registro, control y manejo de las historias clínicas, 7) Modernizar el sistema de gestión de citas médicas y 8) 
Diseñar e implementar el sistema de gestión de seguridad y salud en el trabajo, sobre todo teniendo en cuenta las nuevas condiciones de la pandemia relacionada con la COVID 19.

\section{Componente Tecnológico}

El CAP cuenta con cuatro computadores personales con conexión a internet, uno en la recepción y tres en los consultorios. Usa la hoja de cálculo de Microsoft Excel para el registro de las citas médicas, el control de los horarios del personal médico y el archivo de los resultados de del laboratorio de las pruebas de citología. Las historias clínicas de los pacientes se registran en formatos de papel y se guardan en el archivo físico del CAP. Además, se utiliza el Sistema de Salud Pública SIVIGILA para realizar reportes semanales sobre las enfermedades tratadas en el CAP. En la vista física de la solución tecnológica se documenta las tecnologías necesarias para el despliegue y funcionamiento del sistema de información gerencial diseñado.

\subsection{Solución tecnológica}

La solución tecnológica se presenta exclusivamente para los procesos relacionados con la prevención del VPH. El diseño se documenta bajo la perspectiva $4+1$ (Kruchten, 1995), por eso inicialmente se presenta la vista de escenarios en función de los procesos más importantes del CAP, describiendo el flujo secuencial de las actividades realizarlas en el CAP. Luego se presenta la vista lógica del sistema de información gerencial, explicando cada uno de sus componentes para entender la forma cómo el sistema atiende cada uno de los requisitos establecidos. Finalmente se presenta la vista física con el propósito de especificar las tecnologías necesarias para el despliegue del sistema de información gerencial para el apoyo a la prevención del VPH.

\section{Vista de escenarios}

Para el cumplimiento de su función social relacionada con la detección y prevención del VPH, el CAP realiza tres procesos fundamentales: 1) Atención a los pacientes brindando el servicio de citología, 2) gestión de las campañas preventivas y 3) Gestión del inventario de insumos. Cada uno de estos procesos se describe a continuación. La atención de los pacientes comprende tres momentos, el primero corresponde a la programación de la cita médica, el segundo consiste directamente en la prestación del servicio de citología y el tercero concierne al diagnóstico y la entrega de los resultados. La gestión de las campañas preventivas implica primero hacer un diseño de la campaña, luego una programación y finalmente la realización de la campaña. Por último, la gestión del inventario corresponde al control sobre las entradas y salidas de los insumos bajo el principio FIFO (Firts in firts out), teniendo en cuenta que se trata de productos con fecha de vencimiento. En consecuencia, se diseñó la vista de escenarios representada en Figura 5.

Se definieron tres actores para el sistema. El primero corresponde al paciente, quien puede programar sus citas médicas a partir de la disponibilidad que tenga en su momento el sistema. También tendrá la posibilidad de consultar su historia clínica para conocer los resultados y el diagnóstico de los exámenes ordenados por el personal médico. El segundo actor es el personal médico, quien podrá registrar su disponibilidad de tiempo, para que el sistema genere automáticamente la agenda de citas médicas. De igual forma podrá actualizar la historia clínica cuando atienda al paciente, o cuando genere el diagnóstico a partir de los resultados de los exámenes de citología. Por último, la enfermera tendrá la posibilidad de gestionar las campañas de detección y prevención del $\mathrm{VPH}$, generando el diseño respectivo de la campaña y su programación, además de gestionar el inventario, registrando las entradas y salidas de insumos. 
Figura 5

Vista de escenarios

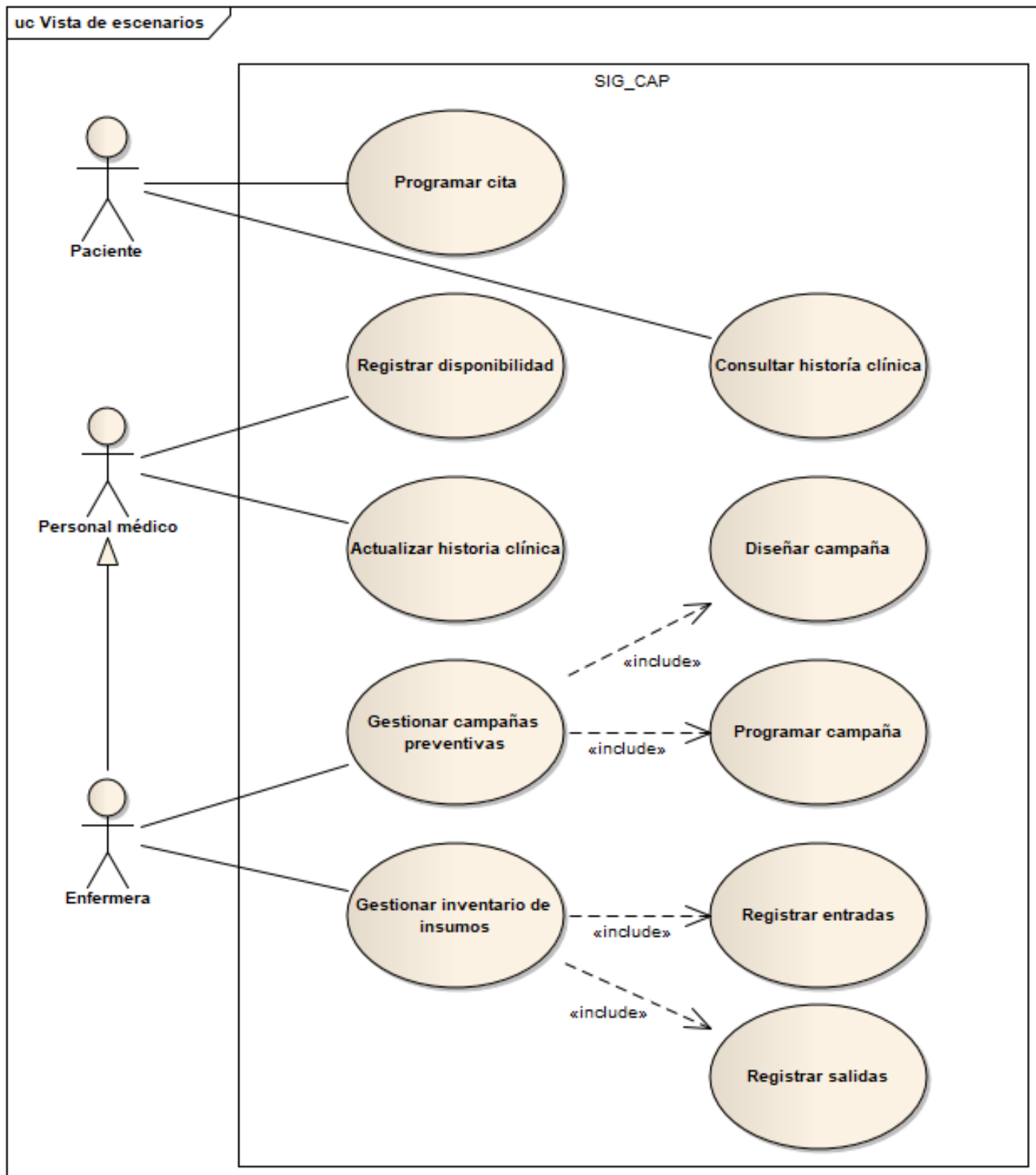

Fuente: De los Autores

\section{Vista lógica}

Para atender los requisitos funcionales del sistema se optó por aplicar una arquitectura limpia (Martin, 2018), garantizando la evolución del sistema en función de la lógica del negocio, sin depender de las tecnologías requeridas para su implementación, como es el caso de las interfaces de usuario para los distintos dispositivos, los motores de bases de datos, etc. En esencia el sistema de información gerencial está estructurado en tres capas, como se observa en Figura 6. 
Figura 6

Vista lógica

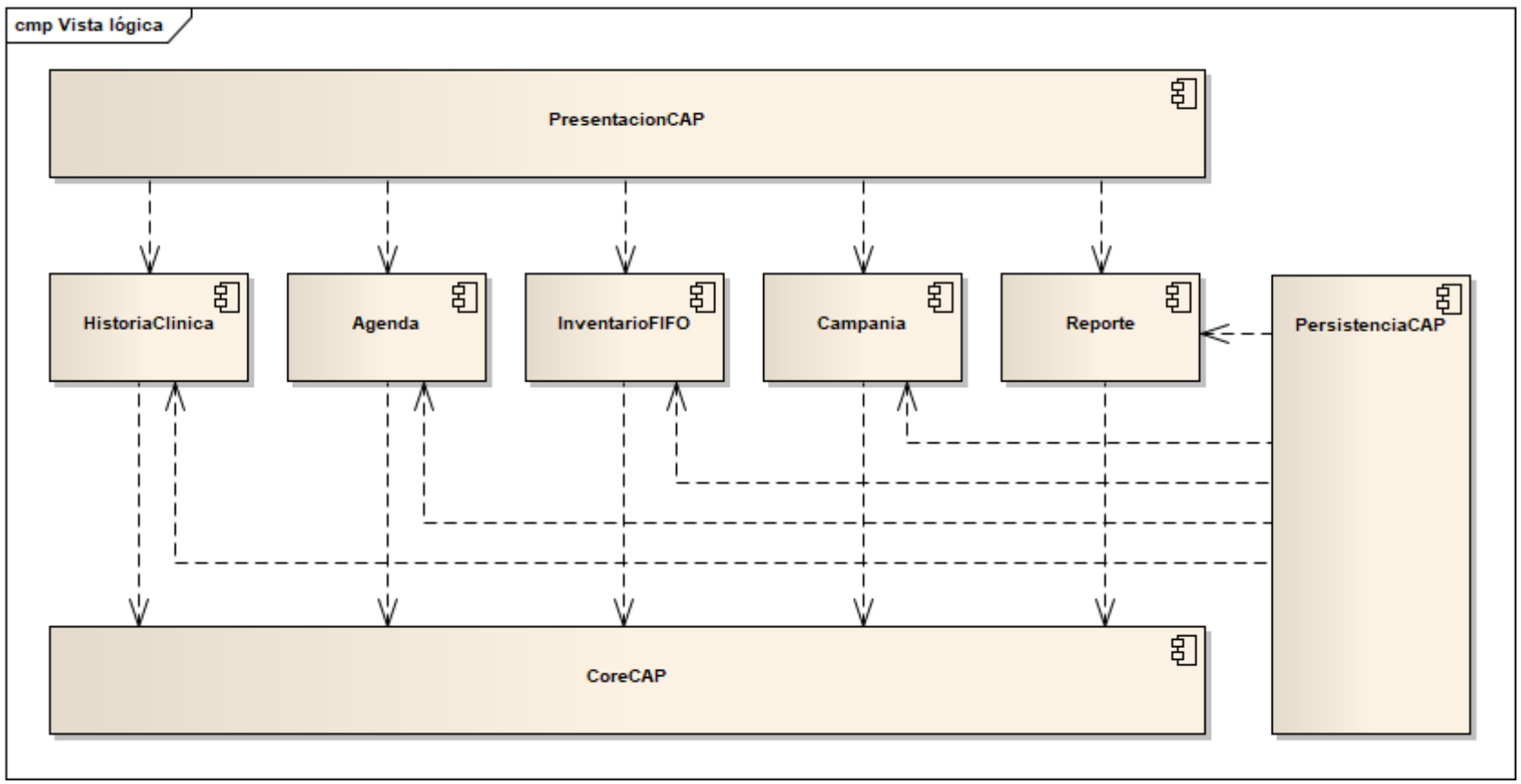

Fuente: De los Autores

El componente CoreCAP corresponde a las entidades del contexto del problema: El personal médico con su estructura jerárquica, el paciente de igual forma con su estructura jerárquica, la historia clínica, los resultados de la citología, la agenda de los servicios prestados, entre otras entidades. La segunda capa está conformada por los componentes responsables de la lógica del negocio y como se observa en el diagrama 4 sólo dependen de las entidades. Cada uno de estos componentes es responsable de funcionalidades establecidas en los requisitos del sistema. El componente de la historia clínica se encarga de la creación, registro y actualización de la historia clínica de cada paciente. Este componente se desarrollará bajo el estándar HL7 para garantizar la compatibilidad con otros sistemas que apliquen el mismo estándar. El componente agenda comprende las funciones relacionadas con la definición y actualización de la disponibilidad del personal médico, así como también de la programación y asignación de las citas médicas. Para el diseño y programación de las campañas de detención y prevención del VPH se diseñó el componente Campania. El control del inventario de los insumos para la prestación de los diferentes servicios es el componente InventarioFIFO. Finalmente, para la generación de los informes que apoyarán la toma de decisiones por parte de la alta gerencia del CAP se diseñó el componente Reporte.

La tercera capa involucra dos aspectos que tradicionalmente se ubican en capas diferentes: La interacción del sistema y su persistencia, pero que bajo el enfoque de arquitecturas limpias corresponden al mismo nivel de abstracción, porque dependen de las tecnologías usadas para su implementación (Martin, 2018). Esta capa se fusionó con la capa del Gateway por la misma razón. Como su nombre lo indica, la capa de presentación será la responsable de garantizar la captura y visualización de los datos y se implementará usando tecnologías con características responsive para no depender de las plataformas tecnológicas del despliegue. Por su parte la capa de persistencia también garantizará la heterogeneidad con respecto a los diferentes motores de bases de datos.

\section{Vista física}

Manteniendo los lineamientos de la arquitectura limpia (Martin, 2018) el despliegue del sistema no depende de tecnologías especializadas, sin embargo, se debe aclarar que la representación de la vista física que se muestra en - Figura 7, debe ser ajustada en función de la implementación que se haga del diseño, en lo referente a uso 
de servidores de aplicaciones y motores de bases de datos. Para el despliegue del sistema se pueden utilizar los equipos que actualmente tiene el CAP.

Figura 7

Vista física

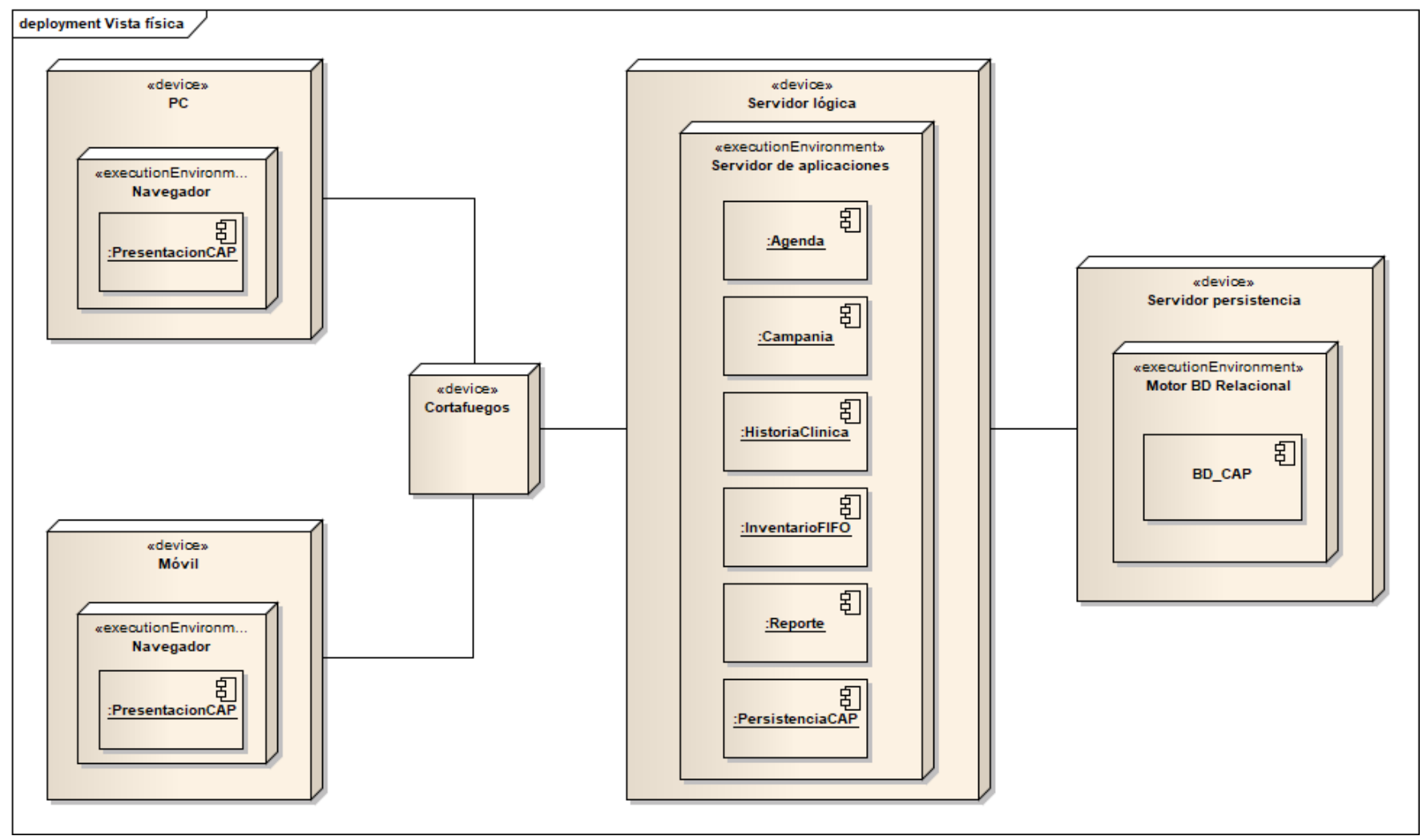

Fuente: De los Autores

Adicionalmente el sistema de información gerencial podrá ser utilizado desde dispositivos móviles de cuarta generación. Para garantizar la seguridad de los datos se requiere de un corta fuegos físico o lógico. Teniendo en cuenta la cantidad de información, el número de transacciones que realiza actualmente el CAP y los requisitos de disponibilidad establecidos, no es necesario implementar medidas de redundancia para garantizar que los servicios estén activos. Si bien se utilizará un motor de bases de datos relacional, gracias al estilo arquitectónico utilizado, el sistema de información gerencial diseñado no dependerá de uno en especial, lo cual le brinda la posibilidad al CAP de valorar otras variables al momento de desplegar el sistema.

\section{Discusiones}

En este trabajo se puede identificar como principal aporte el diseño del sistema de información que generará valor agregado al ser implementado en el Centro de Atención Primaria (CAP) de la Universidad de Cartagena. Por otra parte, el Centro de Atención Primaria (CAP) podrá afrontar retos organizativos y administrativos relacionados con:

- la optimización de la agenda del personal médico, la identificación de programas de formación del personal médico con el fin de mejorar la prestación de servicios,

- la gestión adecuada de insumos para la realización de las pruebas,

- el rediseño de campañas de detección y promoción del VPH para ampliar su cobertura,

- las estrategias para facilitar el registro, control y manejo de las historias clínicas,

- la modernización del sistema de gestión de citas médicas y

- el diseño e implementación del sistema de gestión de seguridad y salud en el trabajo. 
La solución propuesta se describe inicialmente por medio de la vista de escenarios en la que se detallan los procesos y flujos secuenciales de las actividades más relevantes del CAP. Luego se presenta la vista lógica del sistema de información gerencial, en la que se explican los componentes que permiten entender cómo el sistema satisface lo requisitos establecidos. Seguidamente, se explica la vista física en el cual se especifican las tecnologías necesarias para el despliegue de la solución propuesta.

Con este sistema de información gerencial se esperan resultados muy positivos como los obtenidos en la investigación realizada en la institución educativa María Ulises Dávila Pinedo - Morales, donde aplicaron la metodología de sistemas blandos de Peter Checkland como estrategia para la prevención de la infección del VPH (Portocarrero Gutierrez, 2015). Otros trabajos realizados para prevenir el VPH son los tipos de software educativo que utilizan un aspectos psicopedagógicos y enfoque cognitivista-constructivista, con lo que obtuvieron resultados satisfactorios al comparar el antes y después en grupos control y experimentales, demostrando la eficacia del producto software desarrollado en función del conocimiento adquirido (Romero-Vásquez \& López Jiménez, 2020).

Igualmente, se deberá fortalecer la estrategia educativa con pacientes para apoyar el conocimiento sobre el VPH y la prevención del cáncer de cuello uterino (Ventura-Morales et al., 2017). Como se desarrolló en este estudio, se pudo identificar la población que posee bajo nivel conocimiento para focalizar las campañas de capacitación. Finalmente, se recomienda a otros investigadores mejorar la actual propuesta con aspectos como la implementación de algoritmos de clasificación de pacientes u otra técnica inteligente para el análisis de datos que permitan la toma de decisiones especializada. Otro punto a fortalecer es el incremento de los datos históricos recopilados de los servicios prestados en el CAP, ya que no fue posible obtener información sobre otros años diferentes al 2019 debido a que las instalaciones se encontraban cerradas al público por el estado de cuarentena decretado por el Gobierno Nacional de Colombia.

\section{Conclusiones}

Teniendo en cuenta los resultados presentados y su discusión se puede concluir que:

i) La aplicación de la metodología de componentes (Laudon \& Laudon, 2012) sirvió para definir la solución conceptual representada en el diseño de un sistema de información gerencial para apoyar al CAP de la Universidad de Cartagena en su función social relacionada con la prevención del VPH.

ii) Si bien la solución conceptual presentada es construida a partir de la información recolectada en el CAP de la Universidad de Cartagena, su implementación puede ser desplegada en diferentes instituciones de la misma naturaleza, porque está estructurada en función de los componentes organizativo, administrativo y tecnológico.

iii) La solución propuesta no sólo comprende el desarrollo de un producto software, sino que involucra aspectos de carácter organizativo y administrativo, que hace parte de la cultura organizacional.

iv) El CAP de la Universidad de Cartagena afronta dos desafíos principales: 1) Generar un mayor impacto en la comunidad ampliando el número de usuarios atendidos y mejorando la calidad de los servicios que ofrece; y 2 ) Sistematizar sus procesos.

v) El alcance del proyecto sólo corresponde a la solución conceptual y su implementación hace parte de la segunda fase, que comprende el desarrollo del sistema y su despliegue en las Instalaciones del CAP de la Universidad de Cartagena. 


\section{Referencias bibliográficas}

Amit, R. H., Massa, L., \& Zott, C. (2011). The Business Model : Recent Developments and Future Research. Journal of Management, 37(4), 1019-1042.

Bray, F., \& Piñeros, M. (2016). Cancer patterns, trends and projections in latin america and the caribbean: A global context. Salud Publica de Mexico, 58(2), 104-117. https://doi.org/10.21149/spm.v58i2.7779

Contreras-González, R., Magaly-Santana, A., Jiménez-Torres, E., Gallegos-Torres, R., Xeque-Morales, Á., Palomé-Vega, G., García-Aldeco, A., \& Perea-Ortíz, G. (2017). Nivel de conocimientos en adolescentes sobre el virus del papiloma humano. Enfermería Universitaria, 14(2), 104-110.

https://doi.org/10.1016/j.reu.2017.01.002

da Rocha, C. E. de A. L., Marca, Y. P., \& Schneider, F. K. (2018). Support platform for decision-making in research and technological development in public health. Espacios, 30(4), 14-26. https://www.revistaespacios.com/a18v39n06/a18v39n06p14.pdf

de Vries, E., Arroyave, I., \& Pardo, C. (2018). Re-emergence of educational inequalities in cervical cancer mortality, Colombia 1998-2015. Journal of Cancer Policy, 15(December 2017), 37-44. https://doi.org/10.1016/j.jcpo.2017.12.007

de Vries, E., Buitrago, G., Quitian, H., Wiesner, C., \& Castillo, J. S. (2018). Access to cancer care in Colombia, a middle-income country with universal health coverage. Journal of Cancer Policy, 15(January), 104-112. https://doi.org/10.1016/j.jcpo.2018.01.003

Ferlay, J., Soerjomataram, I., Ervik, M., Dikshit, R., Eser, S., Mathers, C., Rebelo, M., Parkin, D. M., Forman, D., \& Bray, F. (2013). GLOBOCAN 2012 v1.0, Cancer Incidence and Mortality Worldwide: IARC CancerBase. No. 11 [Internet]. Lyon, France: IARC.

Guerrero, R., Gallego, A. I., Becerril-Montekio, V., \& Vásquez, J. (2011). Sistema de salud de Colombia. In Salud Publica de Mexico. https://doi.org/10.1590/s0036-36342011000500003

Islam, T., Hutchinson, A. M., \& Bucknall, T. K. (2018). Nurse manager risk information management for decisionmaking: A qualitative analysis. Collegian. https://doi.org/10.1016/j.colegn.2017.10.009

Kruchten, P. (1995). Architecture blueprintsthe 4+1 view model of software architecture. Tutorial Proceedings on TRI-Ada 1991: Ada's Role in Global Markets: Solutions for a Changing Complex World, TRI-Ada 1995, 12(November), 540-555. https://doi.org/10.1145/216591.216611

Laudon, K. C., \& Laudon, J. P. (2012). Sistemas de informacion gerencial. In Climate Change 2013 - The Physical Science Basis. https://doi.org/10.1017/СВ09781107415324.004

Laudon, K. C., \& Laudon, J. P. (2014). Sistemas de información gerencial Decimocuarta edición Decimocuarta edición. Pearson.

Maass, M. C., Asikainen, P., Mäenpää, T., Wanne, O., \& Suominen, T. (2008). Usefulness of a Regional Health Care Information System in primary care. A case study. Computer Methods and Programs in Biomedicine. https://doi.org/10.1016/j.cmpb.2008.04.005

Murillo, R., Luna, J., Gamboa, O., Osorio, E., Bonilla, J., \& Cendales, R. (2010). Cervical cancer screening with naked-eye visual inspection in Colombia. International Journal of Gynecology and Obstetrics, 109(3), 230234. https://doi.org/10.1016/j.ijgo.2010.01.019 
Object Management Group, I. (2015). OMG Unified Modeling Language TM ( OMG UML ), Superstructure. Object Management Group, Inc, 21(March), 758. https://doi.org/10.1007/s002870050092

Piñeros, M., Gamboa, O., Hernández-Suárez, G., Pardo, C., \& Bray, F. (2013). Patterns and trends in cancer mortality in Colombia 1984-2008. Cancer Epidemiology, 37(3), 233-239. https://doi.org/10.1016/j.canep.2013.02.003

Portocarrero Gutierrez, C. E. (2015). Aplicación De La Metodología De Sistemas Blandos De Peter Checkland En La Prevención De La Infección Del Virus Del Papiloma Humano En Los Alumnos De Primer Año De Media Del C.E Nº031 María Ulises Davila Pinedo - Morales. UNIVERSIDAD NACIONAL DE SAN MARTíN - T.

Romero-Vásquez, A., \& López Jiménez, F. (2020). Desarrollo de un software educativo para la orientaciónaprendizaje de la detección del cáncer cervicouterino. Revista Iberoamericana de Investigación y EI Desarrollo Educativo, 11(21).

Tjalma, W. A. A., Kim, E., \& Vandeweyer, K. (2017). The impact on women's health and the cervical cancer screening budget of primary HPV screening with dual-stain cytology triage in Belgium. European Journal of Obstetrics and Gynecology and Reproductive Biology, 212, 171-181.

https://doi.org/10.1016/j.ejogrb.2017.01.010

Ventura-Morales, B., Castellanos-Rojas, M., Chávez Montes de Oca, V. G., \& Sánchez-Valdivieso, E. A. (2017). Estrategia educativa breve para mantenimiento del conocimiento sobre el virus del papiloma humano y prevención del cáncer en adolescentes. Clínica e Investigación En Ginecología y Obstetricia, 44(1), 8-15. https://www.sciencedirect.com/science/article/pii/S0210573X15000660

Vives, A., Cosentino, M., \& Palou, J. (2020). The role of human papilloma virus test in men: First exhaustive review of literature. Actas Urologicas Espanolas, 44(2), 86-93.

https://doi.org/10.1016/j.acuro.2019.08.010

Zhao, Y., Liu, L., Qi, Y., Lou, F., Zhang, J., \& Ma, W. (2020). Evaluation and design of public health information management system for primary health care units based on medical and health information. Journal of Infection and Public Health, 13(4), 491-496. https://doi.org/10.1016/j.jiph.2019.11.004

Esta obra está bajo una Licencia Creative Commons Attribución-NoCommercial 4.0 International

(cc) EY-NC 